\title{
The impact of physical environment on performance and satisfaction in the white collar office: an overview of the literature
}

\author{
Daniel Mittleman \\ Center for the Management of Information \\ The University of Arizona \\ Tucson, AZ 85721 \\ (520) 621-2932 \\ danny@arizona.edu
}

\begin{abstract}
Extended Abstract
Jean Wineman ${ }^{1}$ provided a seminal review of research analyzing the relationship of white collar work environment to job satisfaction and productivity. While previous research had explored the relationship between physical environment and both performance and satisfaction in the industrial workplace, her work was the first summary of findings for that relationship among white collar knowledge workers. This presentation ${ }^{2}$ updates and re-evaluates the status of current research that analyzes the relationship of white collar work environment on job satisfaction and productivity in office environments. This presentation describes the construction of both the independent and dependent measures used by Wineman, reviews recent research organized by Wineman's categories, and broaches the subject of how the physical environment impacts collaboration among knowledge workers.

Work environment is the range of requirements necessary for the health, well being and function of individuals in the workplace. This presentation organizes its evaluation of the work environment into three categories: PHYSICAL COMFORT; SYMBOLIC IDENTIFICATION; and EMERGING ISSUES. PHYSICAL COMFORT includes all factors of the environment which affect the contentment of workers and their ability to carry out work tasks. These factors include the ambient environment, ergonomics, lighting and view, privacy and social interaction, visual and acoustical privacy, and social interaction. SYMBOLIC IDENTIFICATION is the presence of status indicators and perceptions of status in the workplace. Finally several issues are lumped together under the rubric of EMERGING
\end{abstract}

\footnotetext{
${ }^{1}$ Wineman, J. D., 1982, "Office design and evaluation: an overview," Environment and Behavior, 14(5), pp271-298.
}

${ }^{2}$ This presentation stems from: Mittleman, D. D., 1996, Office Design and Evaluation: An Overview Revisited, Working Paper, The University of Arizona. 
ISSUES. Among these are office automation, collaboration, open office planning, control over the physical environment, and methodological concerns.

Satisfaction and performance are measured in several ways. Work environment satisfaction has been measured through survey instruments developed for the construct by several different researchers. While there is reasonable face validity for all of these measures, the lack of a consistent validated measure of construct of satisfaction with the work environment may contribute to differential findings among studies. Job satisfaction is a pleasurable or positive emotional state resulting from the appraisal of one's job or job experiences. Job satisfaction is one of the most established and studied constructs in the organizational psychology literature. While several different instruments have been used to measure job satisfaction in recent studies, most investigators have used well established and validated measures. Work performance is the amount of output produced by a worker. It can either be measured directly by counting output in some manner or perceptually by recording either the worker's own perception of performance or a supervisors perception of the worker's performance. Differing measures of performance is a serious threat to the external validity of many of the studies reviewed. Self measures and perceptual measures of performance may suffer from significant confounds with other perceptual or attitudinal factors.

In this presentation about 50 studies are reviewed and evaluated against the criteria set forth above. The state of the research literature is described. Opportunities for future research on these questions are explored.

Several emerging issues are discussed. They include: OFFICE AUTOMATION, COLLABORATION, OPEN OFFICE PLANNING, CONTROL OVER THE PHYSICAL ENVIRONMENT, and METHODOLOGICAL CONCERNS. Previous reviews of office automation as a moderator for the impact of physical environment impact on performance and satisfaction had occured prior to the ubiquitous arrival of personal computers in the office. Recent widespread usage of PCs has lead to heightened concerns such as VDT radiation and carpel tunnel syndrome. Current computer technology trends include audio, video, and data conferencing. These trends may impact issues such as privacy, social interaction and symbolic identification.

Open office systems planning, an absence of floor-to-ceiling walls and the use of systems furniture to landscape the workplace, was prevalent in the literature in the 1970s and became a common design strategy in the 1980s. Some are beginning to look beyond the basic systems furniture approach to team-based setting supporting group work. Research in the 1980s provided mixed success in its implementation.

Control over the workers physical environment consists of two related constructs. One is the amount of flexibility the environment affords for change to address the needs of an individual worker. Systems furniture, for example, that can be rearranged into different shapes might well afford much greater flexibility than systems furniture assembled so that it must remain in its initial shape. The other construct is the amount of control the worker has over the design or redesign of the work environment. Several studies have been undertaken during the past decade where workers have participated in the design of their workspace. Such a practice is slowly becoming more commonplace.

Wineman noted that relatively little field research has been conducted on behavioral responses to office settings and that the field research that has been conducted has usually been isolated case studies or surveys. However, most of the studies cited in this review have been survey or case studies. Only two are laboratory experiments and none are field experiments. Other advances in research methods are gradually making their way into workplace evaluation research including behavioral mapping and comprehensive post occupancy evaluations. 